\title{
EDITORIAL
}

\section{Nontraditional Epilepsy Treatment Approaches}

Small-molecule antiepileptic drugs (AEDs) have been available since the discovery in 1857 of the anti-seizure properties of bromide. ${ }^{1}$ In the subsequent 150 years, drug treatment has been the foundation of epilepsy therapy and has immeasurably improved the quality of life for many persons with epilepsy. Nevertheless, despite the availability today of more than 25 small-molecule AEDs, many patients do not achieve seizure freedom. All AEDs have side effects that limit their utility, and none is known to be free of teratogenicity. The introduction of more than 10 new AEDs in the last 15 years has not measurably reduced the proportion of patients achieving seizure freedom. ${ }^{2}$ While newer drugs are less likely to impair cognition and behavior, no AED is completely free of such adverse effects. ${ }^{3}$ Moreover, no drug has yet been found that leads to an eradication of epilepsy. A small number of patients benefit from epilepsy surgery, which is an effective alternative form of therapy for selected patients with intractable partial epilepsy. Vagal nerve stimulation may also be helpful for some patients. Still, the currently available therapies do not provide a satisfactory solution for many patients. This has led to frustration and a call for new thinking.

To encourage such innovative thinking, we organized the Fourth Workshop on New Horizons in the Development of Antiepileptic Drugs: Nontraditional Approaches to Treat Epilepsy, which was held at the Clontarf Castle, Dublin, March 5 through 7, 2008. The New Horizons series was founded by Wolfgang Löscher and Dieter Schmidt, with the inaugural workshop held in Philadelphia in 2001'; subsequent workshops were held in Cambridge, Massachusetts $(2003)^{5}$ and Washington, D.C. (2005). ${ }^{6}$ Previous workshops in the series focused on the discovery and development of new small-molecule AEDs, the search for new molecular targets to prevent and cure epilepsy, and strategies to overcome AED resistance. New Horizons IV represented a radical departure. For the first time in the series-and indeed, for the first time in a comprehensive fashion in any venue of which we are awareattention moved completely away from orally delivered small molecules to nontraditional epilepsy therapy approaches.

Our hope in organizing New Horizons IV was to engender disruptive technologies, which are innovations that eventually overturn the existing dominant technology. In a field in which small-molecule AEDs have been the dominant technology for a century and a half, the focus of innovation has been on sustaining technologies: incrementally better small molecules with enhanced performance over established drugs. No doubt researchers have had as their goal the discovery of a revolutionary AED that would control seizure activity in all patients. However, this goal has been elusive, ${ }^{7}$ and it seems unlikely that a "magic bullet" will arise from efforts to identify better small-molecule AEDs. With this in mind, we concluded that an intensive workshop would be useful to focus attention on non-small-molecule therapeutic strategies that had the potential to be disruptive to the established paradigm. New Horizons IV brought together nearly 60 basic epilepsy researchers, neurologists, neurosurgeons, bioengineers, and experts in drug delivery (FIG. 1). The articles in this issue of Neurotherapeutics were prepared by the participants nearly a year after the workshop, during which time they have been able to integrate and reflect on the provocative lectures and lively discussions in Dublin.

The workshop began with an inspiring presentation by Arnold Kriegstein (University of California, San Francisco) on "Building the brain: lessons for the cell therapy of epilepsy." Kreigestein discussed new research on the generation of cellular diversity and cell number in the developing cortex. He proposed that an understanding of these mechanisms will be useful in guiding approaches to generate large numbers of specific neurons for cell-based therapies. Mark Saltzman (Yale University) presented a keynote address at Trinity College on "Nanotechnology for the delivery of drugs to the brain," which discussed the fabrication of drug-loaded nanoparticles from biocompatible materials and their use in the treatment of brain disorders, including epilepsy. Bennewitz and Saltzman (pp. 323336) review the uses of such nanoparticles for epilepsy therapy. The remainder of the workshop was organized into sections on physical approaches, including electrical and magnetic stimulation and cooling (Theme 1), cell therapy with embryonic stem cells and various types of engineered cells (Theme 2), gene therapy with viral vectors (Theme 3 ), novel delivery approaches, including direct delivery into the brain and approaches to non-invasively circumvent the blood-brain barrier (Theme 4), and hormonal and dietary therapies, including botanicals and herbs (Theme 5 ). At the conclusion of the lectures in each section, 


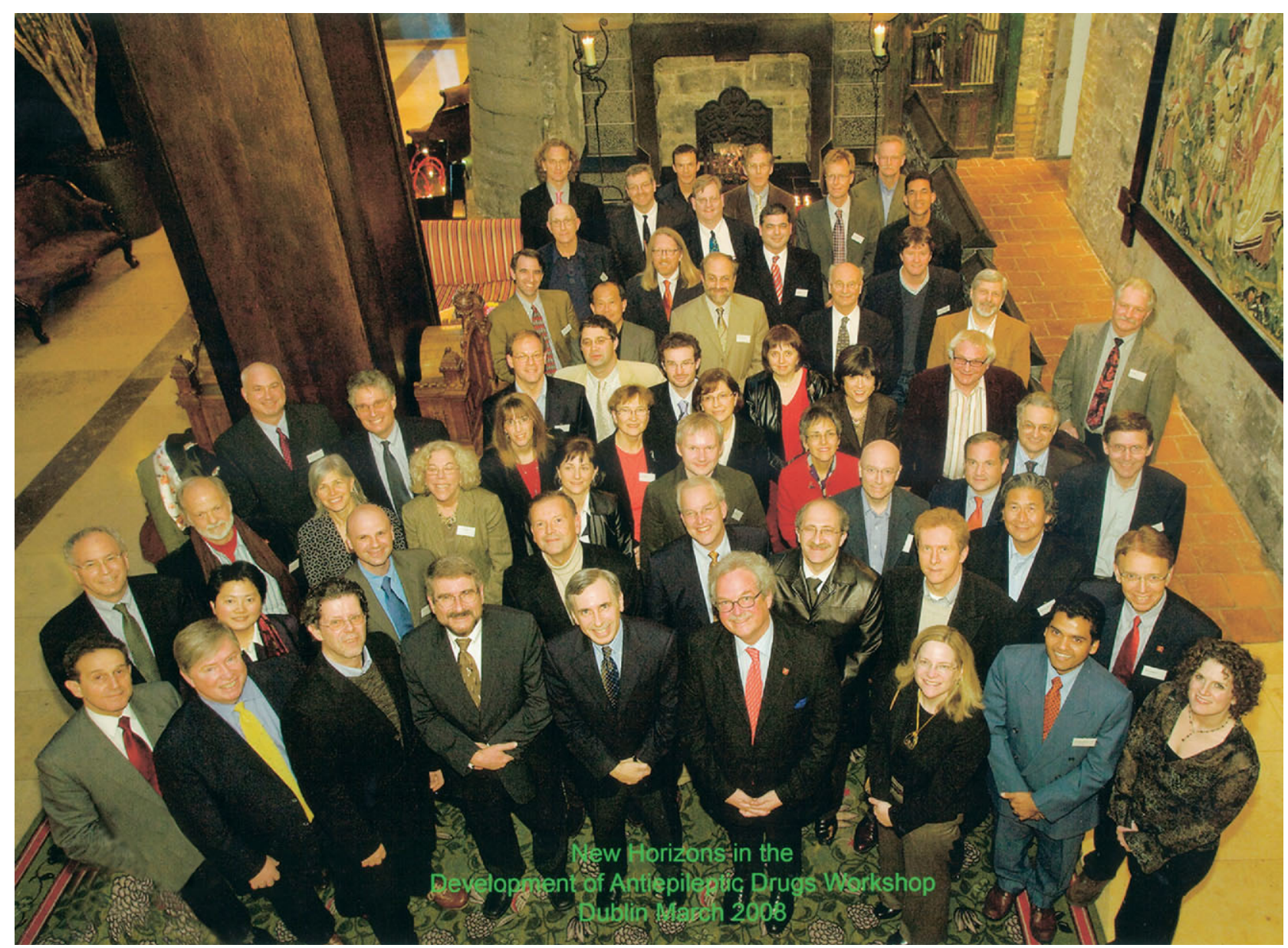

FIG. 1. Participants in the Fourth Workshop on New Horizons in the Development of Antiepileptic Drugs: Non-Traditional Approaches to Treat Epilepsy, at the Clontarf Castle Hotel, Dublin.

two discussants summarized and critically evaluated the therapeutic strategy; one of the discussants commented on its prospects and the other on shortcomings and obstacles. In most cases, the two discussants have collaborated on the commentary articles that are published in conjunction with each theme in this special issue. An overview of the articles in each section is provided below.

\section{THEME 1: BRAIN STIMULATION AND COOLING}

For decades, neurologists have lagged behind cardiologists in the ability to treat disorders of abnormal tissue excitability by electrical stimulation, but this situation is changing, and the future of electrical stimulation for the control of epilepsy is promising. Vagal nerve stimulation (VNS) was approved by the U.S. Food and Drug Administration in 1997 and is also approved in all member countries of the European Union, Canada, Australia, and elsewhere. More than 50,000 persons have been implanted with VNS devices. However, the impact of VNS has been limited.
It is tempting to assume that direct brain stimulation would do better. Since the work of Cooper in the 1970 s $^{8}$ open-label and small, blinded trials have provided evidence supporting the efficacy of deep brain stimulation (DBS) in epilepsy therapy. A recent wellcontrolled, multicenter trial of anterior nucleus of the thalamus stimulation produced statistically significant evidence of seizure reduction. These encouraging results will undoubtedly motivate additional trials to determine whether DBS offers advantages over VNS or other therapeutic options. Boon et al. (pp. 218-227) provide an overview of VNS and DBS, and Milby et al. (pp. 228-237), focusing on VNS, describe the surgical procedure, discuss potential mechanisms, and review the evidence that chronic stimulation reduces the frequency, intensity, and duration of seizures. Skarpaas and Morrell (pp. 238-243) discuss intracranial stimulation in more detail. These authors highlight the distinction between open-loop (scheduled) stimulation, in which stimulation is delivered according to a fixed program, and closed-loop (responsive) stimulation, in which stimulation is contingent upon 
the detection of epileptiform activity. An implantable closed-loop neurostimulator has shown preliminary evidence of efficacy and is currently under investigation in a randomized, controlled trial. Nitsche and Paulus (pp. 244-250) describe the use of repetitive transcranial magnetic stimulation (rTMS) and transcranial direct current stimulation (tDCS). While rTMS has shown promise in animal models, clinical trials have observed weak and variable efficacy. Optimization of the stimulation parameters may improve performance. Tonic tDCS has also shown activity in brain slice and animal models. In one controlled human trial in which anodal excitability-reducing tDCS (1 mA) was applied once for 20 minutes over the epileptic focus, a longterm reduction in seizure frequency was observed. Finally, Rothman (pp. 251-257) describes experimental studies demonstrating that cooling can rapidly terminate seizures in in vitro brain slices and in animals in vivo without causing tissue damage. He discusses the prospect of engineering an implantable system for clinical use containing a solid-state thermoelectric device, and he proposes that the device could be activated by a closed loop seizure recognition system.

\section{THEME 2: CELL THERAPY}

Although experimental studies demonstrating an effect of tissue and cell grafts on seizure susceptibility date to the $1980 \mathrm{~s}$, progress has accelerated in recent years due to advances in stem cell biology and cell engineering. Collectively, the new results discussed in this section engender optimism that practical approaches for the cell therapy of epilepsy may be within reach. Maisano et al. (pp. 263-277) review studies supporting the concept that embryonic stem cell-derived GABAergic precursors could be used to replace hippocampal inhibitory interneurons for the treatment of mesial temporal lobe epilepsy. Boison (pp. 278283) describes an alternative approach for the cell therapy of epilepsy using cells engineered to release the inhibitory neuromodulator adenosine. A lentiviral vector has been constructed that causes persistent knockdown of the adenosine metabolizing enzyme adenosine kinase. Easily accessible human mesenchymal stem cells are treated ex vivo with the viral vector to produce cells that could be used for autologous transplantation, thus diminishing the risk of immune rejection. Epileptic mice receiving transplants of such cells exhibited reduced seizure frequency and duration. Thompson (pp. 284-294) reviews the evidence supporting the use of genetically engineered cells of various types that produce GABA. Baracia and Gallego (pp. 337-343) also discuss cell transplantation strategies. In their commentary, Kriegstein and Pitkänen (pp. 295-299) provide a comprehensive survey of cell therapy research in epilepsy and address the question of how far cell therapy is from clinical application.

\section{THEME 3: GENE THERAPY}

Gene therapy is conventionally viewed as the treatment of a genetic disorder by replacing a defective gene with its normal counterpart. To date, this form of gene therapy has not been attempted in epilepsy. Rather, investigators have developed methods to induce brain cells to constitutively secrete proteins that they expect will cause a local reduction in cellular excitability, an approach that is akin to "hijacking" cells to serve as factories for the local production of an AED. Although lentiviruses and adeno-associated viruses (AAV) are suitable vectors for gene therapy of neural tissues because they persistently transduce non-dividing cells, recent studies in epilepsy have focused on AAV vectors, which elicit minimal immune responses and are nonpathogenic. ${ }^{9}$ Vezzani et al. (pp. 300-306) describe AAV vectors engineered to express the human neuropeptide $\mathrm{Y}$ (NPY) gene. Such vectors have been demonstrated to induce persistent NPY overexpression in the hippocampus and were able to protect against seizures in various epilepsy models, including a model of spontaneous recurrent seizures. McCown (pp. 307-311) has utilized a similar strategy with a vector that expresses galanin. Dudek (pp. 319-322) addresses methodological issues in assessing the efficacy of gene therapy approaches in animal models. He cautions against concluding that any treatment is truly antiepileptogenic (able to prevent the development of the epileptic state) if the therapy had been applied during the induction or development phases of epileptogenesis, particular if the therapy altered seizure activity during these phases. Dudek also raises the concern that gene therapy approaches may not have fewer side effects than traditional AEDs. In theory, gene therapy that is restricted to a defined brain region or cell population could have improved tolerability compared with orally delivered AEDs that are distributed throughout the entire brain. In fact, in a recent study discussed by Vezzani et al., gene therapy targeted to the hippocampus was found to protect against seizures without adverse cognitive effects.

\section{THEME 4: NOVEL DELIVERY APPROACHES}

In addition to gene therapy, there are other epilepsy treatment approaches under investigation that target a therapeutic agent locally to the epileptic zone, so that side effects due to actions on non-epileptic brain regions are avoided. For example, convection-enhanced delivery (CED), as discussed by Rogawski (pp. 344-351), provides a means of delivering anticonvulsant substances in a uniform fashion to a restricted brain re- 
gion. A solution of the therapeutic agent is infused under pressure using an implanted fluid-delivery catheter, thus circumventing the blood-brain barrier and depositing the therapeutic agent directly into the extracellular space. Seizure protection conferred by CED administration of diffusible AEDs dissipates rapidly, whereas prolonged (weeks to months) protection can be obtained following the brief infusion of non-diffusible peptide toxins. Baracia and Gallego discuss intracerebroventricular injection, another means of circumventing the blood-brain barrier. This delivery method minimizes systemic toxicity but does not necessarily result in reduced neurological toxicity compared with systemic delivery.

Wermeling (pp. 352-358) discusses intranasal delivery, mainly of benzodiazepines, for the emergency treatment of seizures when intravenous delivery is not available. Kubek et al. (pp. 359-371) also addresses the use of intranasal delivery, not to enhance the speed of drug action, but rather as a means to circumvent the blood-brain barrier for peptides with anticonvulsant properties, such as thyrotropin-releasing hormone. Remarkably, intranasal delivery may provide a means to target substances to temporal lobe structures. When formulated as nanoparticles, substances deposited in the nasal cavity may enter olfactory neurons and be carried through the cribriform plate to the olfactory bulb where they are then transported to the temporal lobe. Nasally administered nanoparticles may also be carried rapidly into adjacent brain structures by nonneuronal routes (such as via the glands of the olfactory epithelium). Thus, intranasal delivery is a potential approach to deliver substances selectively to brain structures relevant to temporal lobe epilepsy. Kubek also discusses the use of implantable microdisks composed of a surface-eroding biodegradable polymer to locally deliver substances with anti-seizure properties directly into an epileptic focus. Such microdisks can confer seizure protection for long periods of time. Finally, White et al. (pp. 372-380) describe the successful creation of blood-brain barrier permeable analogs of anticonvulsant peptides (related to galanin) that are effective when administered systemically.

\section{THEME 5: HORMONES AND DIET}

An increasing body of evidence supports the concept that hormonal fluctuations can influence the occurrence of seizures. Progress in understanding the neurobiological mechanisms underlying such hormonal effects has been accompanied by increased interest in hormonebased therapies. The major focus to date has been on catamenial (menstrual) seizure exacerbations. Herzog (pp. 383-391) reviews the scientific basis for hormonal treatment of catamenial epilepsy, focusing on the use of progestogens, including progesterone, and summarizes the results of recent clinical trials. Reddy and Rogawski (pp. 392-401) discuss neurosteroid replacement as a treatment for catamenial epilepsy. There is evidence that $\mathrm{GABA}_{\mathrm{A}}$ receptor modulating neurosteroids, including the progesterone-derived neurosteroid allopregnanolone and its synthetic analog ganaxolone, may be particularly well suited to treat perimenstrual seizure exacerbations.

Dietary approaches for the treatment of epilepsypredominantly the high fat, low carbohydrate ketogenic diet-have been in use since the 1920s. Clinical application of the ketogenic diet waned with the ascendancy of AEDs in the 1950s. In recent years, however, there has been a resurgence of interest in the neurobiological mechanisms through which the ketogenic diet influences brain function and in research to assess its clinical efficacy in epilepsy and other conditions. Nylen et al. (pp. 402-405) discuss hypotheses for the anti-seizure activity of the diet based on recent research findings. Several of the proposed mechanisms are plausible, but none has been proven definitively. Kossoff and Rho (pp. 406414) review evidence for the ketogenic diet's clinical effectiveness and raise the possibility that the diet may not only have long-term efficacy when it is adhered to but there may also be persistent benefit after discontinuation. Finally, Schachter (pp. 415-420) reviews herbal therapies, which in the United States are regulated as dietary supplements. Herbal remedies have been in use for thousands of years and are still widely administered for the treatment of epilepsy throughout the world, often in conjunction with AEDs. Efforts are underway to define the efficacy and safety of these substances using modern scientific methods. Research on herbal medicines could provide unexpected insights into new epilepsy treatment strategies.

In conclusion, New Horizons IV provided a view of the impressive scope of research on nontraditional epilepsy treatment strategies. There is remarkable ferment in the field, with some new approaches, such as deep brain stimulation, at advanced stages of clinical development, and several pioneering technologies, including cell and gene therapy, poised to enter the mainstream of epilepsy research. It can be confidently predicted that some of these new approaches will become a routine part clinical practice in coming decades, markedly improving the lives of people with uncontrolled epilepsy. We hope that the articles collected in this special issue promote research toward this worthy goal.

Acknowledgments: We thank the participants for their contributions to both the New Horizons IV workshop and this special issue, UCB Pharma (John-Kenneth Sake and Fan Zhang) for sponsoring the travel and meeting costs, and Huntsworth Health for event planning and onsite support. UCB did not influence the scientific content of the workshop, and neither the organizers nor the participants were paid for their participation. 
Michael A. Rogawski, M.D., Ph.D. Professor of Neurology University of California, Davis School of Medicine Sacramento, California Gregory L. Holmes, M.D. Professor of Neurology and Pediatrics Neuroscience Center at Dartmouth Dartmouth Medical School Lebanon, New Hampshire

\section{REFERENCES}

1. Pearce JM. Bromide, the first effective antiepileptic agent. J Neurol Neurosurg Psychiatry 2002;72:412.

2. Brodie MJ, Kwan P. Staged approach to epilepsy management. Neurology 2002;58(8 Suppl 5):S2-S8.
3. Loring DW, Marino S, Meador KJ. Neuropsychological and behavioral effects of antiepilepsy drugs. Neuropsychol Rev 2007;17:413425.

4. Löscher W, Schmidt D. New horizons in the development of antiepileptic drugs. Epilepsy Res 2002;50:3-16.

5. Löscher W, Schmidt D. New horizons in the development of antiepileptic drugs: the search for new targets. Epilepsy Res 2004;60: 77-159.

6. Löscher W, Schmidt D. New Horizons in the development of antiepileptic drugs: innovative strategies. Epilepsy Res 2006;69:183272.

7. Dichter MA. Innovative clinical trial designs for future antiepileptic drugs. Epilepsia 2007;48(Suppl 1):26-30.

8. Rosenow J, Das K, Rovit RL, Couldwell WT. Irving S. Cooper and his role in intracranial stimulation for movement disorders and epilepsy. Stereotact Funct Neurosurg 2002;78:95-112.

9. Löscher W, Gernert M, Heinemann U. Cell and gene therapies in epilepsy-promising avenues or blind alleys? Trends Neurosci 2008;31:62-73. 